Geofísica Aplicada ao Estudo de Fluxos da Água no Solo Fernando Morais, Luis de Almeida Prado Bacellar

\title{
GEOFÍSICA APLICADA AO ESTUDO DE FLUXOS DA ÁGUA NO SOLO
}

\section{Geophysics Applied to Study of Water Flow in the Soil}

\author{
Fernando Morais \\ Depto. de Geografia - Universidade Federal do Tocantins - Campus de Porto Nacional \\ Porto Nacional/TO - Brasil \\ morais@uft.edu.br \\ Luis de Almeida Prado Bacellar \\ Depto. de Geologia - Universidade Federal de Ouro Preto \\ Ouro Preto/MG - Brasil \\ luisapbacellar@gmail.com
}

Artigo recebido para publicação em 16/09/2009 e aceito para publicação em 21/03/2010

RESUMO: $\quad$ A eletrorresistividade vem sendo bastante aplicada em estudos que buscam compreender como se dáo fluxo de água no solo e consequentemente a recarga de aquiferos. O presente trabalho teve por objetivo avaliar a interferência de algumas propriedades físicas do solo na determinação da frente de saturação predita com o uso da eletrorresistividade e comparar como se dá a percolação na zona não-saturada, observando a variação de perfis de resistividade obtidos em um intervalo de tempo determinado. Os experimentos foram conduzidos em solos da região sudeste do complexo de rochas do embasamento cristalino (Complexo Bação), no interior do Quadrilátero Ferrífero, centro-sul do Estado de Minas Gerais, Brasil. Foram selecionadas duas áreas que se distinguem pelas características geomorfológicas. A primeira, em encosta com formas côncavas em planta e em perfil, com predomínio de argissolos; a segunda, em encosta com forma convexa em planta e perfil, com predomínio de solos bem desenvolvidos (latossolos). Os resultados mostraram que o método da eletrorresistividade se mostrou eficiente para a delimitação da frente de saturação e avaliação da recarga mesmo em se tratando de diferenças sutis nas propriedades hídricas do solo. Foi possível ainda estabelecer que na feição geomorfológica em forma convexa (nose) a percolação se dá de maneira mais rápida que na de forma côncava (hollow).

Palavras Chave: Eletrorresistividade. Infiltração. Percolação. Complexo Bação.

ABSTRACT: The electrical resistivity is common used in studies that intents to understand as occur the flow of water in soil and consequently as occur the groundwater recharge. The main goal of this paper is to evaluate the interference of some physical properties of the ground in the determination of the front of saturation predicted with the use of the electrical resistivity and to compare as if of the a percolating in the vadose zone, observing the variation of gotten profiles of resistivity in an interval of definitive time. Experiments were conducted in soils of southeastern complex crystalline rocks of reliance (Bação complex), within the Quadrilatero Ferrifero, Centro-Southern State of Minas Gerais, Brazil. For this experiment it was chosen two different areas by geomorphologic features. The first one is hollow on the slippery slope, with the predominance of lixisols; the second one is nose on the slippery slope, with well-developed topsoil prevalence (ferralsols). The results showed that the electrical resistivity method proved efficient 
Geofísica Aplicada ao Estudo de Fluxos da Água no Solo

Fernando Morais, Luis de Almeida Prado Bacellar

for the delimitation of saturation and evaluation reloading even when it comes to subtle differences in the properties of soil water. It was also possible to establish that in the nose slope percolation is faster than in the hollow.

Keywords: Electrical resistivity. Infiltration. Percolation. Bação Complex.

\section{INTRODUÇÃO}

Com o advento das políticas ambientais muitos estabelecimentos industriais passaram a ter que apresentar relatórios sobre as condições de qualidade do solo e da água nos locais em que estes se encontram instalados. Por isso há o grande interesse em tornar os levantamentos dos dados que compõem estes relatórios de mais fácil aquisição, sem comprometer sua qualidade.

Dentre os diversos métodos indiretos apropriados ao monitoramento ambiental, a eletrorresistividade vem sendo bastante aplicada em estudos que buscam compreender como é o avanço de plumas de contaminação em aterros sanitários, complexos petroquímicos e cemitérios, dentre outros (LIMA et al., 1995; DEPOUNTIS et al., 2005). Este método é também aplicado à compreensão de como se dá o fluxo de água na zona não-saturada do solo (DAILY et al., 1992; PARK, 1998; HAGREY e MICHAELSEN, 1999; MUKHTAR et al., 2000; NEVES, 2002; ACWORTH et al., 2005), isso porque a resistividade dos solos normalmente diminui com o aumento da umidade ou do conteúdo de sólidos dissolvidos na água intersticial (NASCIMENTO et al. 1999).

Segundo Orellana (1972) à dificuldade que um corpo apresenta em se deixar atravessar por uma corrente elétrica dá-se o nome de resistividade elétrica ou eletrorresistividade, como é mais comumente referida na literatura. Esta propriedade baseia-se na lei de Ohm, pela qual, tendo-se conhecimento da corrente injetada $(\mathrm{I})$, da diferença de potencial $(\Delta \mathrm{V})$ e das posições dos pontos de injeção e captação de correntes (eletrodos de corrente e potencial respectivamente), pode-se calcular a resistividade utilizando-se a equação:

$\rho=(\Delta \mathrm{V} / \mathrm{I}) \cdot \mathrm{K}(\Omega . \mathrm{m})$

onde,

$\mathrm{K}=2 \pi \cdot \mathrm{Gx}$

sendo:

$\mathrm{G}=1 /[(1 / \mathrm{n})-(2 / \mathrm{n}+1)+(1 / \mathrm{n}+2)$

onde, $\mathrm{K}$ é um fator geométrico para o arranjo dipolodipólo que depende da forma de disposição dos eletrodos de corrente (AB) e potencial (MN) no terreno; $x$ é o espaçamento dos dipolos $\mathrm{AB}$ e $\mathrm{MN}$ adotados; e "n" é o nível ou profundidade investigada.

A equação 1 baseia-se na aplicação de cargas elétricas em um terreno homogêneo e isotrópico, ou seja, qualquer que fosse a disposição dos eletrodos, estes estariam localizados em pontos isorresistivos. Porém, tal fato não é encontrado na natureza, assim, na realidade os valores obtidos representam uma resistividade aparente $\left(\rho_{\mathrm{a}}\right)$ (ORELLANA, 1972).

Um problema observado em trabalhos que estudam plumas de contaminação a partir de levantamentos geofísicos deve-se ao fato desses adotarem dados regionais das características hídricas do solo. Na maioria dos casos esses dados não representam as propriedades locais, tendo em vista que a condutividade hidráulica é uma propriedade que apresenta grande variabilidade espacial.

O presente trabalho teve por objetivo avaliar a interferência de algumas propriedades físicas do solo na determinação da frente de saturação predita 
Geofísica Aplicada ao Estudo de Fluxos da Água no Solo Fernando Morais, Luis de Almeida Prado Bacellar

com o uso da eletrorresistividade. Procurou-se ainda comparar como se dá a percolação na zona não-saturada, observando a variação de perfis de resistividade obtidos em um intervalo de tempo determinado. Finalmente, procurou-se testar a hipótese proposta por Morais e Bacellar (2006), os quais propõem para as áreas estudadas, que em feições convexas do relevo a recarga tende a ocorrer de forma mais rápida que nas formas côncavas.

\section{2. ÁREA DE ESTUDO}

Os experimentos foram conduzidos em solos da região sudeste do complexo de rochas do embasamento cristalino (Complexo Bação), no interior do Quadrilátero Ferrífero, entre as cidades de Ouro Preto e Belo Horizonte, no centro-sul do Estado de Minas Gerais. Foram selecionadas duas áreas, uma próxima ao colégio Dom Bosco e outra próxima ao córrego Holanda. Nessas áreas já foram realizados estudos sobre a pedogênese, a evolução geomorfológica, as características geotécnicas e conservação dos solos. Na região o clima é do tipo Cwa, de Koppen, com marcante contraste pluviométrico anual. As chuvas concentram-se nos meses de outubro a março, com uma média anual de $1.348 \mathrm{~mm}$ e temperaturas médias anuais de $19^{\circ} \mathrm{C}$ (SANTOS, 2001), apresentando evapotranspiração média mensal de 74 mm (PARZANESE, 1991).

A geologia das áreas é caracterizada por rochas gnáissicas finamente bandadas, de composição predominantemente granodiorítica (DOOR, 1969), com manto de intemperismo normalmente muito espesso, alcançando localmente até 50 metros de espessura.

O relevo é suave, com colinas e morrotes com pequenos desnivelamentos de terreno, inferiores a $70 \mathrm{~m}$ (BACELLAR, 2000). De acordo com os modelos de encostas propostos por Ruhe (1975), a área denominada estação Dom Bosco pode ser caracterizada como uma encosta de forma côncava em planta e em perfil, também conhecida como hollow ou concavidade, enquanto a estação Holanda caracteriza-se como uma encosta de forma convexa em planta e em perfil, denominada nose ou saliência.

A região apresenta vegetação primária dominada por campos cerrados, atualmente substituídos por pastagens muito degradadas, como na estação Holanda, e medianamente degradadas, como na Estação Dom Bosco (FARIAS, 1992).

Nos setores de relevo mais plano da região predominam solos bem desenvolvidos (latossolos vermelho-amarelos), enquanto que nos trechos mais íngremes ou geodinamicamente mais jovens predominam argissolos e cambissolos (PARZANESE, 1991; BACELLAR, 2000).

Os horizontes pedológicos superficiais (A e B) exibem textura argilo-arenosa e, mais raramente, areno-argilosa (SOBREIRA, 2000; SILVA, 2000; BACELLAR, 2000; FUTAI, 2002). Eles mostram razões silte/argila variando entre 0,10 e 1,3 (BACELLAR, 2000; FUTAI, 2002; MORAIS, 2003; MORAIS e BACELLAR, 2006), faixa típica de solos bem evoluídos, como os latossolos (RESENDE et al., 1995).

O horizonte $\mathrm{B}$ apresenta espessuras que variam normalmente entre 1 e $10 \mathrm{~m}$ e contém basicamente quartzo, caulinita e gibbsita (PARZANESE, 1991; MORAIS e BACELLAR, 2006), secundados por goethita, illita, mica, sílica e óxido de alumínio amorfo e Fe livre (FUTAI, 2002).

A textura desses solos em ensaios granulométricos sem uso de defloculante é arenosa, desprovida de argila, devido à forte agregação (PARZANESE, 1991; BACELLAR, 2000; SILVA, 2000; FUTAI, 2002). Estudos químicos e micromorfológicos apontam que parte das frações areia e silte é constituída por pseudomorfos de caulinita. Por tal razão, estes solos apresentam elevados índices de estabilidade de agregados (PARZANESE, 1991; BACELLAR, 2000).

Morais e Bacellar (2006) realizaram ensaios com infiltrômetro de anéis concêntricos, 
permeâmetro Guelph e permeâmetro de carga constante e observaram valores médios de condutividade hidráulica de $2,20 \times 10^{-5} \mathrm{~m} / \mathrm{s}$ e 2,09 x $10^{-5} \mathrm{~m} / \mathrm{s}$ para as profundidades de $0,15 \mathrm{~m}$ e $0,50 \mathrm{~m}$ respectivamente, na estação Holanda. Na estação Dom Bosco os valores médios encontrados foram de $1,44 \times 10^{-5} \mathrm{~m} / \mathrm{s}$ e $6,70 \times 10^{-6} \mathrm{~m} / \mathrm{s}$ para $0,15 \mathrm{~m} \mathrm{e}$ $0,50 \mathrm{~m}$ de profundidade, indicando que, nesta região, a recarga se dá de forma mais lenta nas concavidades que nas partes convexas do relevo. Para os autores, tais diferenças podem ser explicadas pelo fato da estação Holanda apresentar solos pedologicamente mais desenvolvidos (latossolos) que aqueles encontrados na estação Dom Bosco (argissolos).

O saprolito (horizonte $\mathrm{C}$ ), que apresenta espessuras de até 40 metros (SOBREIRA, 2000), é composto essencialmente por quartzo, feldspato (variavelmente alterado para caulinita), illita e muscovita (PARZANESE, 1991; FUTAI, 2002; MORAIS, 2003).

\section{METODOLOGIA}

Para a realização deste estudo, foram selecionadas duas áreas, com características pedogeomórficas e de conservação distintas (MORAIS e BACELLAR, 2006), denominadas por Bacellar (2000) como estações Dom Bosco e Holanda.

Com base nas investigações realizadas por Morais e Bacellar (2006), foram escolhidos pontos com valores de condutividade hidráulica contrastantes, sendo 2 na estação Dom Bosco (DB10 e DB27) e 2 na estação Holanda (H03 e H18). Com uso de um cylinder infiltrometer (HILLS, 1970) foram injetados 20 litros de solução salina saturada de $\mathrm{NaCl}$ no ponto DB27 e 40 litros nos demais pontos.

Nestes pontos foram realizados levantamentos de eletrorresistividade com o uso de um aparelho que trabalha sob o principio de corrente contínua, por isto foram utilizados eletrodos nãopolarizáveis. Para a realização destes levantamentos foi estabelecida uma linha de 13 metros, na qual o ponto de injeção da solução salina foi localizado no centro da linha. A disposição dos eletrodos obedeceu à configuração do arranjo dipólo-dipólo com $1 \mathrm{~m}$ de espaçamento entre os eletrodos, totalizando 5 níveis de investigação (FIGURA 1).

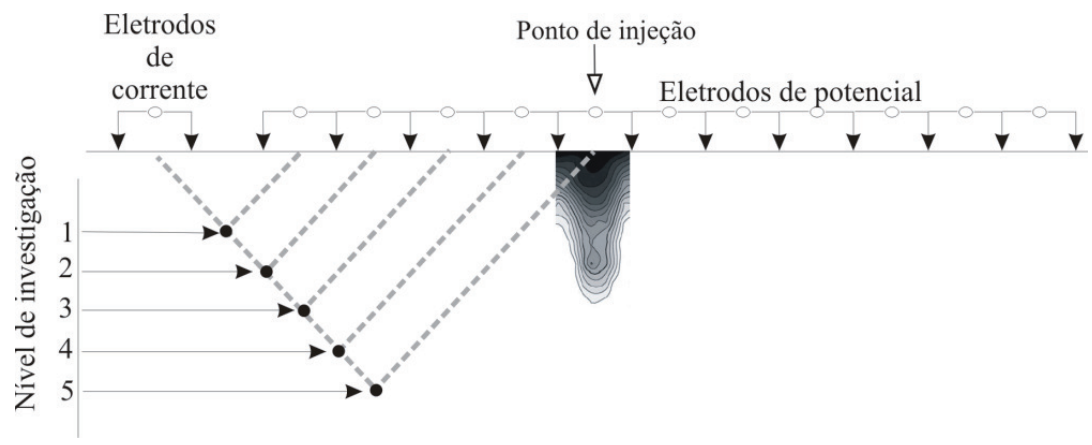

FIGURA 1: Configuração dos eletrodos dispostos no arranjo dipólo-dipólo com ponto de injeção da solução salina.

Em cada ponto foi feito um caminhamento antes da injeção da solução salina, a fim de ter-se um conhecimento dos valores de resistividade elétrica do solo em condições naturais (background), além de servir de comparação com valores subsequentes. Depois da injeção da solução salina foram obtidos perfis espaçados no tempo, sendo o primeiro uma hora após o inicio da injeção de solução. Os demais perfis foram distribuídos no seguinte intervalo de tempo:

- no ponto H03, foram feitos levantamentos 
Geofísica Aplicada ao Estudo de Fluxos da Água no Solo Fernando Morais, Luis de Almeida Prado Bacellar

$\operatorname{nos} 1^{\circ}, 2^{\circ}, 5^{\circ}, 7^{\circ}, 9^{\circ}, 12^{\circ}$ e $27^{\circ}$ dias de ensaio;

- no ponto $\mathrm{H} 18, \operatorname{nos} 1^{\circ}, 2^{\circ}, 5^{\circ}, 7^{\circ}, 9^{\circ}, 12^{\circ}$ e $27^{\circ}$

dias de ensaio;

- no ponto DB10, nos $1^{\circ}, 2^{\circ}, 4^{\circ}, 6^{\circ}, 9^{\circ}, 12^{\circ} \mathrm{e}$

$27^{\circ}$ dias de ensaio;

- no ponto DB27, nos $1^{\circ}, 2^{\circ}, 5^{\circ}, 7^{\circ}, 9^{\circ}, 12^{\circ} \mathrm{e}$

$27^{\circ}$ dias de ensaio.

Os valores de resistividade aparente, obtidos em campo, foram interpretados automaticamente utilizando-se do software Res2dinv que tem como finalidade, transformar em quantitativo, o posicionamento qualitativo fornecido pelas seções pseudo-profundidades (pseudo-depth section).

\section{RESULTADOS E DISCUSSÃO}

As resistividades elétricas, medidas antes da injeção da solução salina, permitiram identificar valores de background do terreno que compreendem aos intervalos entre $21-9931,482-1628,122-16100$ e 3796 - 11181 ohm.m. para os pontos DB10, DB27, H3 e H18, respectivamente (FIGURAS 2 e 3). Assim, valores abaixo dos encontrados desses backgrounds representariam anomalias possivelmente associadas à passagem ou presença da solução salina injetada, pois quanto maior a umidade e quantidade de íons na água intersticial, mais condutivo se tornam os solos (TABBAGH et al., 2000). Loke e Barker (1996) mostram que a partir de 5 interações o percentual de erro tende a estabilizar-se; dessa forma os dados aqui apresentados foram trabalhados com 7 iterações.

O ponto DB27 (FIGURA 2) apresentou os menores valores de resistividade; acredita-se que tal comportamento deve-se à sua posição na parte baixa da concavidade, área de convergência de fluxos, apresentando menor condutividade hidráulica e maior umidade natural, contribuindo para melhor condução da corrente elétrica. Os valores de baixa resistividade observados para o quinto nível de investigação desse ponto correspondem à posição do freático, pouco profundo na área.

Os maiores valores de resistividade encontrados a partir do terceiro nível de investigação no ponto H03 são explicados pela considerável abundância de fragmentos de rocha (solo saprolítico), que segundo Elis e Zuquette (2001) contribuem para o aumento da resistividade. Estes valores corroboram os valores típicos de resistividade elétricas dos gnaisses que variam de $6,8 \times 10^{4}$ a $3,0 \times 10^{6}$ de acordo com a umidade (TELFORD et al., 1990), visto que saprolitos tendem a apresentar menores resistividades que a rocha sã, pois apresentam maior umidade. Tal fato já havia sido observado por Bacellar (2000) que realizou ensaios de eletrorresistividade e GPR na área, observando que a rocha (gnaisse) situa-se bem próxima à superfície. $\mathrm{O}$ mesmo autor relacionou os valores de resistividade menores que $6.000 \Omega$.m aos solos saprolíticos da área, enquanto valores superiores a estes, foram associados ao topo do embasamento cristalino na estação Holanda.

Os perfis de resistividades expostos nas FIGURAS 2 e 3 apresentam uma evolução da percolação da solução salina injetada exatamente no centro da linha do caminhamento $(6,5 \mathrm{~m})$ para as estações Dom Bosco e Holanda, respectivamente. Nota-se que o avanço da solução se deu de maneira mais pronunciada nos pontos da estação Holanda, isso poderia ser creditado às características geotécnicas dos solos dessas áreas que, de acordo com Morais e Bacellar (2006), apresentam maior condutividade hidráulica que os solos da estação Dom Bosco. Os valores relacionados à solução infiltrada são aqueles encontrados entre 6 e 7 metros da linha do caminhamento, sendo $<1.500,<1.003$, $<2.416$ e $<3.266 \Omega$.m para os pontos DB10, DB27, $\mathrm{H} 03$ e H18, respectivamente.

Na tentativa de melhor explicar variações entres os pontos da mesma estação pode-se destacar primeiramente a variação dos pontos da estação Dom Bosco que apresentam uma notável diferença nos perfis de infiltração do ponto DB27 se comparados com os referentes ao ponto DB10, sendo que neste ultimo é mais fácil identificar a percolação da solução salina ao longo dos dias. Enquanto no ponto DB27, essa percolação se mostrou de forma mais discreta. Outra consideração que pode ser feita refere-se 
aos valores de resistividade superiores a 1.528 $\Omega . m$, observados entre o segundo e quarto níveis de investigação no ponto DB27, que podem ser creditados à presença de uma camada de solo menos permeável (horizontes $\mathrm{AB}$ e $\mathrm{Bt}$ ) localizada a partir de $0,5 \mathrm{~m}$ de profundidade, como observaram Morais e Bacellar (2006). Estes autores observaram que essa camada possui menor porosidade que o restante do perfil; assim, a menor quantidade de água intersticial torna tal horizonte mais resistivo.

No tocante a diferença das resistividades entre os pontos da estação Holanda, como ressaltado anteriormente, os valores superiores para o ponto H03 estão relacionados à delgada camada de solo que este apresenta, ao contrário do ponto H18 que apresenta solo com espessura mais significativa.

As partir dos valores de baixa resistividade referentes à percolação da solução salina através do solo, pode-se notar que tal fluxo se deu de maneira mais rápida nos solos da estação Holanda que na estação Dom Bosco. Essas variações corroboram e reforçam a hipótese levantada por Morais e Bacellar (2006) de que nas áreas de relevo côncavo (estação Dom Bosco) a recarga tende a se dá de maneira mais lenta que naquelas de forma convexa (estação Holanda). Vale a pena lembrar que os sítios estudados mostram valores de condutividade hidráulica diferentes, porém sutis.

Assim como em outros estudos (HAGREY e MICHAELSEN, 1999; NEVES, 2002), neste trabalho o método da eletrorresistividade se mostrou eficaz para estudos de recarga e fluxos de água na zona vadosa. Ressalta-se ainda que, em função da pequena quantidade de eletrodos disponível, neste trabalho os eletrodos foram retirados ao final de cada ensaio e recolocados no mesmo local para o levantamento subsequente. Entretanto os resultados mostram que o fato de retirar os eletrodos não interferiu de forma a comprometer os resultados obtidos. 
Geofísica Aplicada ao Estudo de Fluxos da Água no Solo Fernando Morais, Luis de Almeida Prado Bacellar

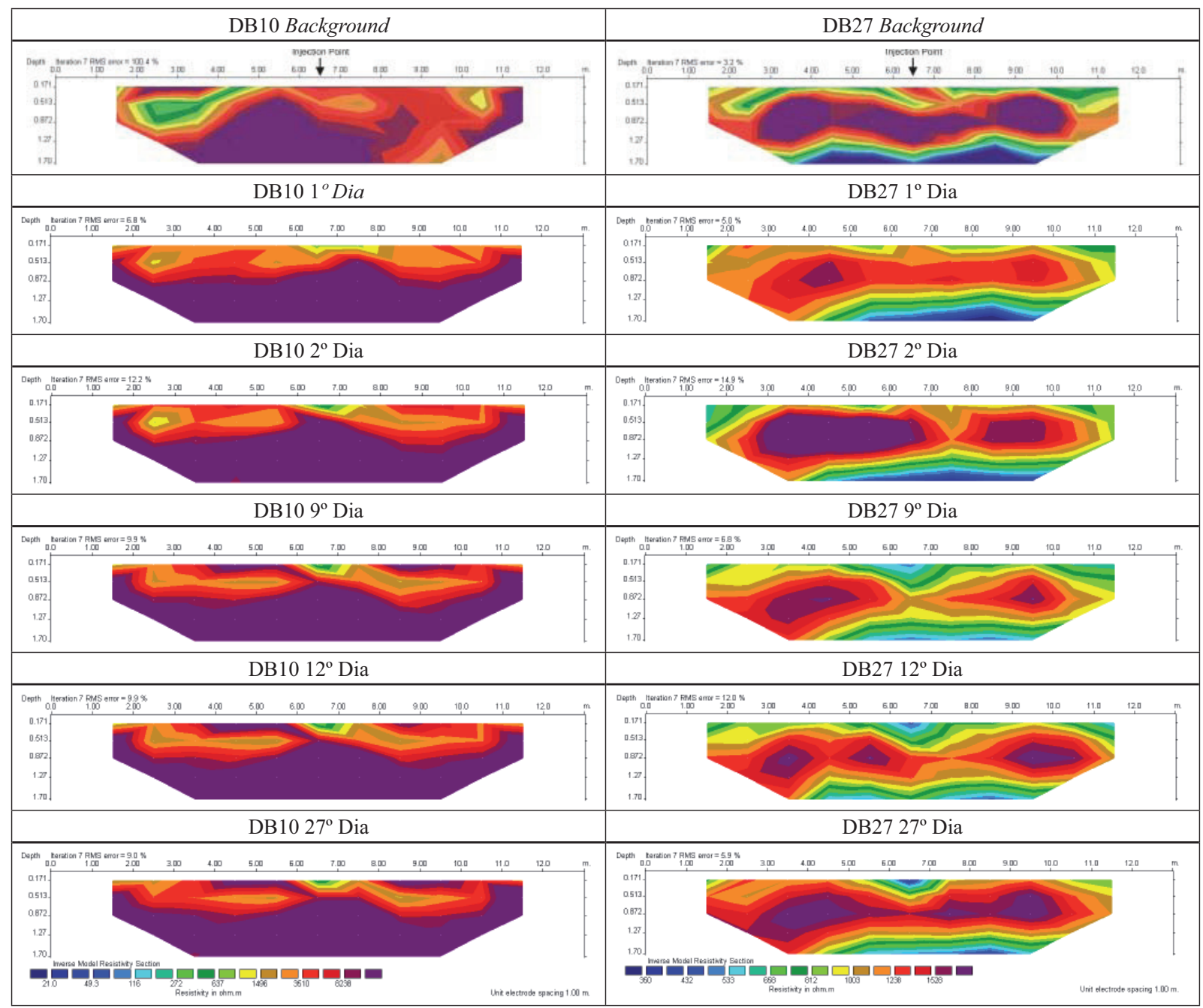

FIGURA 2: Evolução da percolação, estação Dom Bosco. 
Geofísica Aplicada ao Estudo de Fluxos da Água no Solo

Fernando Morais, Luis de Almeida Prado Bacellar

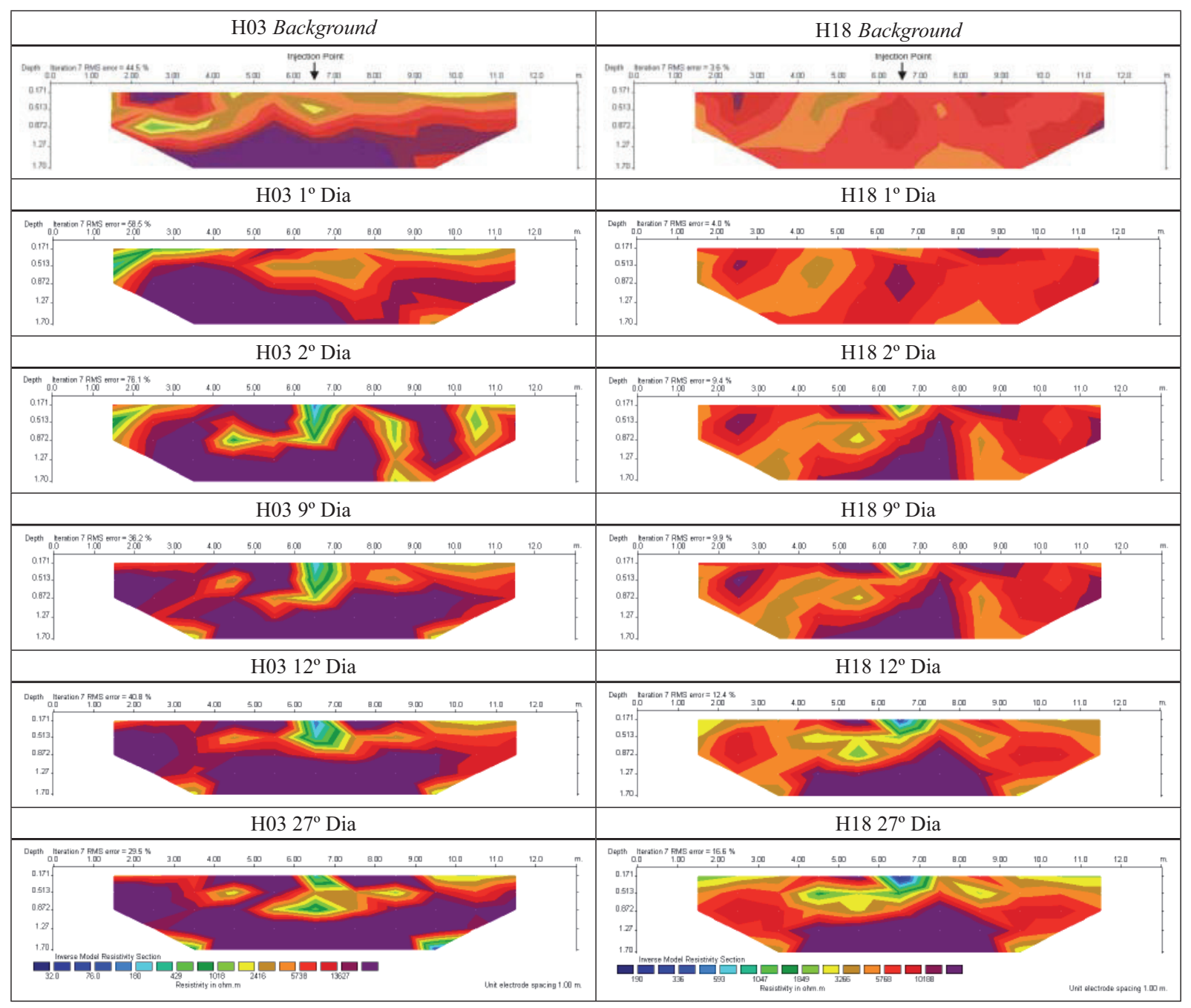

FIGURA 3: Evolução da percolação, estação Holanda.

\section{CONCLUSÕES}

Com o objetivo de avaliar como se dá a percolação na zona não-saturada, neste trabalho foram observados perfis de resistividade elétrica obtidos em um intervalo de 27 dias para cada ponto das duas áreas estudadas. A partir dos resultados obtidos, foi possível estabelecer que na feição geomorfológica em forma convexa (nose) a percolação se dá de maneira mais rápida que na de forma côncava (hollow).

O método da eletrorresistividade se mostrou eficiente para a delimitação da frente de saturação e avaliação da recarga mesmo em se tratando de diferenças sutis nas propriedades hídricas do solo.

\section{REFERÊNCIAS}

ACWORTH, R.I.; YOUNG, R.R.; BERNADI, A.L. Monitoring soil moisture status in a Black Vertosol on the Liverpool Plains, NSW, using a combination of neutron scattering and electrical image methods. Australian Journal of Soil Research, 43, 2005. p. 105 $-117$. 
Geofísica Aplicada ao Estudo de Fluxos da Água no Solo Fernando Morais, Luis de Almeida Prado Bacellar

BACELLAR, L.A.P. Condicionantes Geológicos, Geomorfológicos e geotécnicos dos mecanismos de voçorocamento na bacia do Rio Maracujá, 2000, 225 f. Tese (Doutorado) COPPE/UFRJ, Ouro Preto/ MG, Rio de Janeiro/RJ. 2000.

DAILY, W.; RAMIREZ, A.; LABRECQUE, D.; NITAO, J. Electrical resistivity tomography of vadose water moviment. Water Resource Research, 28(5), 1992. p. $1429-1442$.

DEPOUNTIS, N.; HARRIS, C.; DAVIES, M.C.R.; KOUKIS, G.; SABATAKAKIS, N. Application of electrical imaging to leachate plume evolution studies under in-situ and model conditions. Environmental Geology, 47, 2005. p. $907-914$.

DOOR, J.N. Physiographic and structural development of the Quadrilátero Ferrífero, Minas Gerais. U.S. Geological Survey Professional Paper, 641 -A, 1969.

ELIS, V.R.; ZUQUETTE, L.V. Aplicação integrada de métodos geoelétricos em áreas de disposição de resíduos industriais: cidade de Franca, Brasil. Geotecnia, 92, 2001. p. $47-68$.

FARIAS, C.A. Dinâmica da revegetação natural de voçorocas na região de Cachoeira do Campo, MG. 1992, 63f. Dissertação (Mestrado) - UFV, Viçosa, 1992.

FUTAI, M.M. Estudo teórico-experimental do comportamento de solos tropicais não-saturados: Aplicação a um caso de voçorocamento. 2002, 559f. Tese (Doutorado) - COPPE/UFRJ, Rio de Janeiro, 2002.

HAGREY, S.A.; MICHAELSEN, J. Resistivity and percolation study of preferential flow vadose zone at Bokhorst, Germany. Geophysics, 64(3), 1999. p. 746 -753 .

HILLS, R.C. The determination of the infiltration capacity of fields soils using the cylinder infiltrometer. British Geomorphological Research Group, Technical Bulletin, 3, 1970. p. $1-25$.
LIMA, O.A.L.; SATO, H.K.; PORSANI, M.J. Imaging industrial contaminant plumes with resistivity techniques. Journal of Applied Geophysics, 34, 1995. p.93- 108.

LOKE, M.H.; BARKER, R.D. Rapid least-squares inversion of apparent resistivity pseudosections by a quasi-Newton method. Geophysical Prospecting, 44, p. 131 - 152. 1996.

MORAIS, F. Estudo dos Processos Erosivos Subsuperficiais na Bacia do Rio Maracujá - MG. 2003, 98f. Dissertação (Mestrado) - UFOP, Ouro Preto, 2003.

MORAIS, F. BACELLAR, L.A.P. Análise dos fatores intervenientes na condutividade hidráulica dos horizontes superficiais de solos de áreas gnáissicas do complexo Bação - MG. Solos e Rochas, 29(1), 2006. p. $89-101$.

MUKHTAR, A.L.; SULAIMAN, W.N.; IBRAHIM, S.; LATIF, P.A.; HANAFI, M.M. Detection of groundwater pollution using resistivity imaging at seri petaling landfill Malaysia. Journal of Environmental Hydrology, 8, 2000. p. 1 - 7.

NASCIMENTO, C.T.C.; KOIDE, S.; PIRES, A.C.B.; MELLO, G.A. Pseudo-seções elétricas na avaliação da contaminação do subsolo. Revista Brasileira de Geociências, 29(4), 1999. p. 621 - 626.

NEVES, A.P.V. Estudo geofísico para a caracterização geológica e hidrogeológica da subsuperfície rasa em área de deposição de rejeitos sólidos no município de Barcarena - PA. 2002, 50f. Dissertação (Mestrado) - UFPA, Belém, 2002.

ORELLANA, E. Prospeccion geoelectrica en corriente continua. Ed. Paraninfo, 1972.

PARK, S. Fluid migration in the vadosa zone from 3-D inversion of resistivity monitoring data. Geophysics, 63(1), 1998. p. $41-51$.

PARZANESE, G.A.C. Gênese e desenvolvimento 
das voçorocas em solos originados de rochas granitóides da região de Cachoeira do Campo, Minas Gerais. 1991, 117f. Dissertação (Mestrado) - UFV, Viçosa, 1991.

RESENDE, M.; CURI, N.; REZENDE, S.B.; CORREA, G.F. Pedologia: base para distinção de ambientes. Ed. NEPUT, 1995.

RUHE, R.V. Geomorphology. Houghton Mifflin, 1975.

SANTOS, C.A. Comportamento Hidrológico Superficial, Subsuperficial e a Erodibilidade dos solos da região de Santo Antônio do Leite, distrito de Ouro Preto - MG. 2001, 108f. Dissertação (Mestrado) - UFOP/EM/DEGEO, Ouro Preto, 2001.

SILVA, T.R.M. Caracterização e erodibilidade dos solos de uma voçoroca na região de Ouro Preto MG. 2000. 106f. Dissertação (Mestrado) - PEC COPPE/UFRJ, Rio de Janeiro, 2000.

SOBREIRA, F.G. Processos erosivos acelerados (voçorocas): O exemplo de Cachoeira do Campo, Ouro Preto, MG. Solos e Rochas, 23(3), 2000. p. 217 -233 .

TABBAGH, A.; DABAS, M.; HESSE, A.; PANISSOD, C. Soil resistivity: a non-invasive tool to map soil structure horizonation. Geoderma, 97, 2000. p. $393-404$.

TELFORD, W.M., GELDART, L.P.; SHERIFF, R.E. Applied Geophysics. Cambridge University Press, 1990. 